\title{
Twelve-month clinical outcomes of transradial coronary artery intervention: comparison of the right and left radial artery approach
}

\author{
Corresponding author: \\ Norvydas Zapustas \\ Lithuanian University \\ of Health Sciences \\ Tel. +37063915533 \\ E-mail: norvydaszapustas@gmail.com
}

\begin{abstract}
Introduction. Trans-radial intervention has several advantages such as reduction of bleeding risk, improvement of patients' convenience, and immediate ambulation as compared with the trans-femoral intervention. In the trans-radial intervention, there are some anatomical and technical differences between right and left radial approach. The aim of this study is to evaluate the impact of the choice of the right or left radial approach on 12-month clinical outcomes in patients undergoing the trans-radial intervention.

Methods. A total of 506 consecutive patients who underwent trans-radial intervention were enrolled from Nov 2013 to Oct 2014 in the Lithuanian University of Health Sciences Trans-radial Intervention Registry. The patients were divided into two groups; a right radial approach group and a left radial approach group. To adjust potential confounders, propensity score-matched analysis was performed using the logistic regression model.

Results. After propensity score matching, the baseline clinical and angiographic characteristics were balanced between the two groups. However, contrast volumes during the procedure were larger and fluoroscopic times were longer in the right radial approach group, whereas procedure times were longer in the left approach group. Procedural and in-hospital complications were similar between the two groups. The cumulative clinical outcomes up to 12 months, including mortality, recurrent myocardial infarction, repeat revascularisation, stent thrombosis, and MACE, were similar between the two groups.

Conclusions. In this study, despite the procedural efficacy including procedural time and contrast volume were increased in the right artery approach, however, 12-month cumulative clinical outcomes were similar between the two groups.
\end{abstract}

Key words: percutaneous coronary intervention, trans-radial, right approach, left approach

Med Res J 2016; 1 (3): 108-110
Volume 1, Number 3, 108-110 10.5603/MRJ.2016.0019

Copyright (C) 2016 Via Medica ISSN 2451-2591 
Table 1. Comparison between right and left radial access

\begin{tabular}{lcc}
\hline & Right radial access & Left radial access \\
\hline Acceptability & Widely adopted & Less popular \\
Preparation and setup & More standardised & May be cumbersome \\
Comfort for the operator & ++++ & + \\
Learning curve & Longer & Shorter \\
Catheter manipulation & More technically challenging & Better control \\
Radiation & Similar to left radial access among expert & Shorter with trainees \\
Efficacy and safety & operators & Similar \\
\hline
\end{tabular}

left radial approach was not widely adopted, however, perhaps because it relocated the operator to the left side of the patient and disrupted the traditional laboratory setup. The right radial approach was utilised in the first description of transradial PCl in 1993, as described by Ferdinand Kiemeneij [3]. During this time, dedicated radial catheterisation kits with 22 -gauge access needles were already commercially available, as were the 6-F guiding catheters used for $\mathrm{PCl}$.

The right radial approach has since become the vascular access site of choice for the majority of transradial operators. The familiarity and ease of catheter and equipment manipulation from the right side by both the operator and the ancillary staff, like that of the standard transfemoral route, has probably driven this trend. However, the left radial artery route offers a few advantages over the right radial approach, such that mastery of this technique has become almost compulsory for the modern transradial operator. Furthermore, modifications in patient preparation and equipment setup make it possible to perform left radial catheterisation from the right side of the patient, providing convenience that is comparable to that of right radial or transfemoral access. Table 1 summarises the differences and similarities between right and left transradial access.

\section{Methods}

A total of 506 consecutive patients who underwent transradial intervention (TRI) were enrolled from Nov 2013 to Oct 2014 in the Lithuanian University of Health Sciences TRI Registry. The patients were divided into two groups: a right radial approach group ( $n=240 \mathrm{pts}$ ) and a left radial approach group ( $n=266$ pts). To adjust potential confounders, propensity score-matched (PSM) analysis was performed using the logistic regression model (C-statistics: 0.726). After PSM, a total of 450 patients (225 pairs) were enrolled for this analysis.

\section{Results}

After PSM, the baseline clinical and angiographic characteristics were balanced between the two groups. However, contrast volumes during the procedure were larger and fluoroscopic times (20.5 \pm 26.0 minutes vs. $15.1 \pm 10.6$ minutes $)$ were longer in the right radial approach group $(256.3 \pm 116.6$ cc vs. $225.0 \pm 88.7 \mathrm{cc}, \mathrm{p}$-value $<0.001)$, whereas procedure times $(45.2 \pm 27.4$ minutes vs. $53.4 \pm 25.7$ minutes, $p$-value $=0.003$ ) were longer in the left approach group. After PSM, procedural and in-hospital complications were similar between the two groups. The cumulative clinical outcomes up to 12 months, including mortality, recurrent myocardial infarction (MI), repeat revascularisation, stent thrombosis, and MACE, were similar between the two groups (Tab. 2).

The safety and efficacy of the right and left radial approaches were compared in a recent randomised trial involving 1000 patients who underwent transradial coronary angiography. The study found significantly shorter fluoroscopy times with the left radial approach. This may be partly explained by a three-fold higher incidence in subclavian tortuosity, as well as a higher incidence of radial loops with right radial access. Nevertheless, the overall procedural success rate, total procedure duration, number of catheters used to complete the procedure, and amount of contrast material used were the same between the two routes [4]. A similar observation was seen in patients undergoing transradial $\mathrm{PCl}$ for acute myocardial infarction. In a retrospective series of 135 patients, no significant difference between right and left radial approaches was found in terms of procedure success rate, procedure duration, room-toballoon time, and safety profile [5].

In the hands of expert transradial operators, outcomes are comparable with either right or left radial access. During the learning phase, however, transradial catheterisation is technically demanding and time-con- 
Table 2. Clinical outcomes

\begin{tabular}{lccc}
\hline & $\begin{array}{c}\text { Right radial } \\
(\mathbf{n = 2 2 5 )}\end{array}$ & $\begin{array}{c}\text { Left radial } \\
(\mathbf{n}=\mathbf{2 2 5})\end{array}$ & P value \\
\hline Mortality & $9(4 \%)$ & $11(4.8 \%)$ & $>0.05$ \\
Cardiac death & $7(3.1 \%)$ & $8(3.5 \%)$ & $>0.05$ \\
Recurrent myocardial infarction & $8(3.5 \%)$ & $10(4.4 \%)$ & $>0.05$ \\
Repeat percutaneous coronary intervention & $18(8 \%)$ & $21(9.3 \%)$ & $>0.05$ \\
Stent thrombosis & $3(1.3 \%)$ & $1(0.4 \%)$ & $>0.05$ \\
MACE (mortality, repeat PCl, MI) & $35(15 \%)$ & $42(18 \%)$ & \\
\hline
\end{tabular}

MACE - major adverse cardiac events; $\mathrm{PCl}$ — percutaneous coronary intervention; $\mathrm{Ml}$ - myocardial infarction

suming. Data suggest that it may be advantageous to emphasise the left radial approach during the training period because it appears to allow novice operators to acquire the skills and confidence required for transradial procedures more quickly than the right radial route. This was demonstrated by the TALENT (Left Versus Right Transradial Approach for Percutaneous Coronary Procedures) trial, which randomised nearly 1500 patients undergoing transradial coronary angiography with either right or left radial routes. The study found that among trainees, the left radial approach was associated with a significantly shorter learning curve, with progressive reductions in cannulation and fluoroscopy times as the operator volume increased, compared to right radial access [6].

This difference may be explained by the anatomical variations between the right and left vasculature. In addition to the higher incidence of loops in the right radial artery, the right subclavian artery is often more tortuous than the left, especially in patients with short stature and those who are elderly [7]. Additionally, in the right radial route, the catheter has to pass not only through the right subclavian artery, but also through the brachiocephalic trunk, before reaching the aortic root. Traversing these two areas of bifurcation increases the technical difficulty, especially if atherosclerosis is involved. Because the left subclavian artery arises directly from the aorta, the path followed by the catheter in the left radial route is very similar to that of the transfemoral approach, resulting in less complex catheter manipulation and greater control. Furthermore, the left radial approach provides direct access to the left internal mammary artery (LIMA), which is of special significance in patients who have undergone coronary artery bypass grafting. Certainly, the LIMA can also be cannulated from the right radial route, but it is significantly more challenging from a technical standpoint, and there is a potential risk of embolic stroke due to catheter manipulation in the aortic arch.

\section{Conclusions}

In this study, despite the procedural efficacy including procedural time and contrast volume were increased in right artery approach, however, 12-month cumulative clinical outcomes were similar between the two groups; the right and left routes to transradial catheterisation are equally safe and effective.

Conflict of interest: none declared

\section{References}

1. Rao SV, Cohen MG, Kandzari DE et al. The transradial approach to percutaneous coronary intervention: historical perspective, current concepts, and future directions. J Am Coll Cardiol 2010; 55: 2187-2195.

2. Campeau L. Percutaneous radial artery approach for coronary angiography. Cathet Cardiovasc Diagn 1989; 16: 3-7.

3. Kiemeneij F, Laarman GJ. Percutaneous transradial artery approach for coronary stent implantation. Cathet Cardiovasc Diagn 1993; 30: 173-178.

4. Norgaz T, Gorgulu S, Dagdelen S. Arterial anatomic variations and its influence on transradial coronary procedural outcome. J Interv Cardiol 2012; 25: 418-424.

5. Larsen P, Shah S, Waxman S et al. Comparison of procedural times, success rates, and safety between left versus right radial arterial access in primary percutaneous coronary intervention for acute ST-segment elevation myocardial infarction. Catheter Cardiovasc Interv 2011; 78: 38-44.

6. Sciahbasi A, Romagnoli E, Burzotta F et al. Transradial approach (left vs right) and procedural times during percutaneous coronary procedures: TALENT study. Am Heart J 2011; 161: 172-179.

7. Dehghani P, Mohammad A, Bajaj R, et al. Mechanism and predictors of failed transradial approach for percutaneous coronary interventions. JACC Cardiovasc Interv 2009; 2: 1057-1064. 